\title{
Electron scattering disintegration processes on light nuclei in covariant approach
}

\author{
P.E. Kuznietsov, Yu. A. Kasatkin and V. F. Klepikov
}

Institute of Electrophysics and Radiation Technologies NAS of Ukraine, Kharkiv, Ukraine

\begin{abstract}
We provide general analysis of electro-break up process of compound scalar system. We use covariant approach with conserved EM current, which gives the ability to include strong interaction into QED. Therefore, we receive the ability to describe disintegration processes on nonlocal matter fields applying standard Feynman rules of QED. Inclusion of phase exponent into wave function receives a physical sense while we deal with the dominance of strong interaction in the process. We apply Green's function (GF) formalism to describe disintegration processes. Generalized gauge invariant electro-break up process amplitude is considered. One is a sum of traditional pole series and the regular part. We explore the deposits of regular part of amplitude, and its physical sense. A transition from virtual to real photon considered in photon point limit . The general analysis for electro-break up process of component scalar system is given. Precisely conserved nuclear electromagnetic currents at arbitrary square of transited momentum are received. The only undefined quantity in theory is vertex function. Therefore, we have the opportunity to describe electron scattering processes taking into account minimal necessary set of parameters.
\end{abstract}

\section{Introduction}

Gauge arbitrariness can be pulled off in QED [1]. It can be made by inclusion of generalized Minkowskii space supplemented by "charged" tangent space 
[2]. This operation is made on the basis of universality principle of EM interaction and inseparability property of mass and charge for fundamental particles.

We use the rule of "parallel transition" to implement these statements. This regulation (correlation into the same world point) gives the ability to compare different charged matter fields. It means, mathematically, that covariant derivative of the field function must equal zero in the direction of tangent space. Namely, the additional "charged" coordinate $\psi_{c h}(x)$ introduces into consideration. $\psi_{c h}(x)$ must fulfill the equation:

$$
\left.\frac{d x_{\mu}}{d \tau} D^{\mu} \psi_{c h}(x)\right|_{x=x(\tau)}=\left.\frac{d x_{\mu}}{d \tau}\left(\partial^{\mu}-i e A^{\mu}\right) \psi_{c h}(x)\right|_{x=x(\tau)}=0,
$$

where $\tau$ - natural parameter of trajectory $x_{\mu}(\tau)$ length, $e$ - electric charge, $A^{\mu}$ - vector-potential of external EM field. Solution of equation (1), accounting initial condition $\psi_{c h}(a)=1$, is:

$$
\psi_{c h}(x)=P e^{i e \int_{a}^{x} A^{\nu}(\xi) d \xi_{\nu}},
$$

where $P$ - space-time regulation operator lengthwise trajectory $x_{\mu}(\tau)$.

Total wave function is defined by the product of space-time and "charged" component in generalized configuration space:

$$
\Psi(x ; A)=\psi_{c h}(x) \psi(x)=P e^{i e \int_{a}^{x} A^{\nu}(\xi) d \xi_{\nu}} \psi(x),
$$

The basis of the theory construction are 2-point and 3-point GF. Their structure satisfies the inseparability and indifference properties. Equations for nonlocal 2-point and 3-point GF (taking into account the geometrical interpretation of gauge field) are:

$$
\begin{gathered}
D_{\text {nonlocal }}(x, y ; A)=i<P\left(\phi(x) e^{i e \int_{y}^{x} A^{\nu}(\xi) d \xi_{\nu}} \phi^{+}(y)\right)> \\
G(x, y, z ; A)=<P\left(\phi(z) \times e^{i e_{1} \int_{x}^{z} A^{\nu}(r) d r_{\nu}} \phi_{1}^{+}(x) e^{i e_{2} \int_{y}^{z} A^{\sigma}(r) d r_{\sigma}} \phi_{2}^{+}(y)\right)>
\end{gathered}
$$

\section{Electro-breakup amplitude limit in photon point}

We define gauge-invariant amplitude to provide the opportunity to describe electron scattering processes. This amplitude corresponds to disintegration process of nonlocal scalar field into two scalar fragments [2]. Such amplitude 


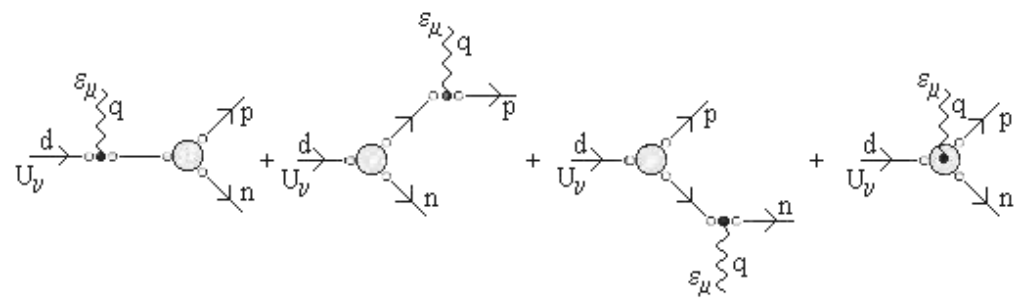

Figure 1: Gauge invariant electro-break up amplitude.

has the shape as in fig. 1. To be precise, we consider scalar deuteron, which consists of two scalar nucleons, which have the value of masses and charges corresponding to real particles. Therefore, matrix element is:

$$
\begin{gathered}
M=e \varepsilon_{\mu} J^{\mu} \\
e=\sqrt{4 \pi \alpha} \\
J^{\mu}=J_{p o l}^{\mu}+J_{r e g}^{\mu} \\
J_{p o l}^{\mu}=z_{s} G_{s} \frac{\left(d+d^{\prime}\right)^{\mu}}{s-m_{d}^{2}}+z_{t} G_{t} \frac{\left(p+p^{\prime}\right)^{\mu}}{t-m_{p}^{2}}+z_{u} G_{u} \frac{\left(n+n^{\prime}\right)^{\mu}}{u-m_{n}^{2}} \\
J_{r e g}^{\mu}=\frac{k^{\mu}}{k q}\left(z_{t} G_{t}+z_{u} G_{u}-z_{s} G_{s}\right)
\end{gathered}
$$

where $\alpha=1 / 137, z_{s, t, u}$ - charges of scalar deuteron, proton and neutron in elementary charge units, $k_{\mu}$ - relative space-like 4 -momentum of $p, n$ pair, $k=(0, \vec{p})$.

The final expression for amplitudes $J_{r e g}^{\mu}$ regular part does not contain a singularity, when proton and neutron are scattered at right angle $\left(\theta=\frac{\pi}{2}\right)$. We consider $\lim _{q k_{s} \rightarrow 0} J_{r e g}^{\mu}$ to show this. We factorize the expression using Taylor functional expansion at the point $x=-k_{s}^{2}$. Thus, we receive that the expression is defined by the derivative of vertex function $G\left(-k_{s}^{2}\right)$ of strong interaction due to the charge conservation law $z_{s}=z_{t}+z_{u}$ :

$\lim _{q k_{s} \rightarrow 0} \frac{\left(z_{t}+z_{u}\right) G\left(-k_{s}^{2}\right)-z_{t} G\left(-k_{s}^{2}+q k_{s}\right)-z_{u} G\left(-k_{s}^{2}-q k_{s}\right)}{q k_{s}}=\left.\lambda \frac{d G(x)}{d x}\right|_{x=-k_{s}^{2}}$ 
We consider the limit of intersection from virtual to the real photon $\left(q^{2} \rightarrow 0\right)$ to show that nonlocal current is conserved. All calculations performed in center of mass framework, where virtual photon momentum oriented along $o Z$ axes. The problem can be separated into three identical parts. They depend from the momentums in $s, t, u-$ channels appropriately. The limit of the amplitude in $s$ - channel is given by the sum of pole part and the third summand in expression (10). We take into account the relation between momentums $q+d=d^{\prime}$ (fig. 1) and expression for Mandelstam variable $s=(d+q)^{2}=m_{d}^{2}+2 d q+q^{2}$. Therefore, the limit of $s-$ channel part can be written down as:

$$
\lim _{q^{2} \rightarrow 0} q_{\mu}\left(J_{\text {spol }}^{\mu}+J_{\text {sreg }}^{\mu}\right)=z_{s} G(s) \lim _{q^{2} \rightarrow 0}\left(\frac{2 q_{\mu} d^{\mu}}{2 d q}-\frac{q_{\mu} k^{\mu}}{k q}\right)=0
$$

In a reciprocal manner one can receive the expressions for $t, u$ channels. Well then, we satisfy the $J_{\text {total }}^{\mu}$ conservation requirement. This property provides the opportunity to describe electro break-up processes applying developed covariant approach.

\section{Conclusions}

The main notice of the article is the description of interaction between electromagnetic field and bound system of strongly interacting particles. Regular part of gauge invariant amplitude defines the value of electrical manyparticle mechanisms dynamical deposit in addition to one-particle (pole) deposit. This statement is in precise agreement with the requirement of gauge invariance. Therefore, in developed covariant approach, the photon point limit of the electro-break up amplitude exists and equals to zero. This property gives the opportunity of unified description of photo and electro processes based on general principles. Application of this approach permits us to receive precisely conserved nuclear electromagnetic currents at arbitrary $q^{2}$.

\section{References}

[1] G.V. Efimov Problems of quantum theory of nonlocal interaction, Moscow, "Science", Head revision of p.-m. literature, 1985, 216p. (in Russian).

[2] Yu.A. Kasatkin. Additional requierements for consistent description of Nonlocal interactions in QED // Physics of Particles and Nuclei letters 2010, Vol.7 N2, p.100-109. 